\title{
Value of serum glycated albumin and high-sensitivity C-reactive protein levels in the prediction of presence of coronary artery disease in patients with type 2 diabetes
}

\author{
Li Jin $\mathrm{Pu}^{\dagger}$, Lin $\mathrm{Lu}^{\dagger}$, Xue Wei Xu, Rui Yan Zhang, Qi Zhang, Jian Sheng Zhang, \\ Jian Hu, Zheng Kun Yang, Feng Hua Ding, Qiu Jin Chen, Sheng Lou, \\ Jie Shen, Dan Hong Fang and Wei Feng Shen*
}

Address: Department of Cardiology, Institute of Cardiovascular Diseases, Rui Jin Hospital, Jiaotong Univerisity Medical School, Shanghai, People's Republic of China

Email: Li Jin Pu - Plj476@yahoo.com.cn; Lin Lu - Lulinshyzl@hotmail.com.cn; Xue Wei Xu - XXW519@sina.com.cn;

Rui Yan Zhang - zry.2630@yahoo.com.cn; Qi Zhang - Zhangqi@yahoo.com.cn; Jian Sheng Zhang - ZJS1942@yahoo.com.cn;

Jian Hu - hujian@medmail.com.cn; Zheng Kun Yang - yangzhenkun2002@hotmai.com; Feng Hua Ding - dingding-1976@yahoo.com.cn;

Qiu Jin Chen - cqj2003@hotmail.com; Sheng Lou - Lousheng-123@163.com; Jie Shen - albenshen@yahoo.com.cn;

Dan Hong Fang - fangdanhong@sohu.com; Wei Feng Shen* - rjshenweifeng@yahoo.com.cn

* Corresponding author †Equal contributors

Published: 20 December 2006

Cardiovascular Diabetology 2006, 5:27 doi:10.1 186/1475-2840-5-27

This article is available from: http://www.cardiab.com/content/5/I/27

(C) 2006 Pu et al; licensee BioMed Central Ltd.

This is an Open Access article distributed under the terms of the Creative Commons Attribution License (http://creativecommons.org/licenses/by/2.0), which permits unrestricted use, distribution, and reproduction in any medium, provided the original work is properly cited.
Received: 22 November 2006

Accepted: 20 December 2006

\begin{abstract}
Background: Coronary artery disease (CAD) is a major vascular complication of diabetes mellitus and reveals high mortality. Up to $30 \%$ of diabetic patients with myocardial ischemia remain asymptomatic and are associated with worse prognosis compared to non-diabetic counterpart, which warrants routine screening for CAD in diabetic population. The purpose of this study was to evaluate the clinical value of serum glycated albumin and high-sensitivity C-reactive protein (hs-CRP) levels in predicting the presence of CAD in patients with type 2 diabetes.
\end{abstract}

Methods: Three hundred and twenty-four patients with type 2 diabetes were divided into two groups based on presence (CAD group, $n=24 \mathrm{I}$ ) or absence (control group, $n=83$ ) of angiographically-documented CAD (lumen diameter narrowing $\geq 70 \%$ ). Serum levels of glycated albumin and hs-CRP as well as serum concentrations of glucose, lipids, creatinine, blood urea nitrogen and uric acid were measured in both groups. Predictors of CAD were determined using multivariate logistic regression model and receiver-operating characteristic (ROC) curves.

Results: Serum glycated albumin and hs-CRP levels were significantly increased in diabetic patients with CAD. Multivariate regression analysis revealed that male gender, age, serum levels of glycated albumin, hs-CRP, creatinine and lipoprotein (a) were independent predictors for CAD. Areas under the curve of glycated albumin and hs-CRP and for regression model were 0.654 (95\% Cl 0.579-0.730, $\mathrm{P}<0.00 \mathrm{I}), 0.72 \mathrm{I}(95 \% \mathrm{Cl} 0.658-0.785, \mathrm{P}<0.00 \mathrm{I})$ and $0.824(95 \% \mathrm{Cl} 0.768-0.879, \mathrm{P}<0.00 \mathrm{I})$, respectively. The optimal values of cut-off point were $18.7 \%$ (sensitivity $67.9 \%$, specificity $60.0 \%$ ) for glycated albumin and $5.2 \mathrm{mg} / \mathrm{l}$ (sensitivity $72.2 \%$, specificity $60.0 \%$ ) for hs-CRP to predict CAD. Logistic regression model was defined as: $\mathrm{P} /(\mathrm{I}-\mathrm{P})=\operatorname{EXP}(-1.5+1.265$ gender +0.812 age +1.24 glycated albumin +0.953 hs-CRP +0.902 lipoprotein(a) +1.918 creatinine). The optimal probability value for predicting CAD in type 2 diabetic patients was 0.648 (sensitivity $82.3 \%$, specificity $68.6 \%$ ).

Conclusion: Serum glycated albumin and hs-CRP levels were significantly elevated in patients with type 2 diabetes and CAD. The logistic regression model incorporating with glycated albumin, hs-CRP and other major risk factors of atherosclerosis may be useful for screening CAD in patients with type 2 diabetes. 


\section{Background}

Coronary artery disease (CAD) is a major vascular complication in patients with type 2 diabetes. Previous studies have shown that up to $30 \%$ of the diabetic patients with CAD had silent ischemia and experienced poor outcome following acute coronary events [1], indicating clinical importance of screening asymptomatic CAD in diabetic population. Advanced glycated end products (AGEs) have been shown to form non-enzymatically in hyperglycemic environment and to be implicated in diabetic vascular complications. Recent studies indicated that AGEs-mediated inflammation is closely related to atherosclerosis process in patients with diabetes [2,3]. Glycated albumin is a predominant glycated protein in vivo and exerts adverse effects on vascular biological function. Determination of serum glycated albumin level provides more information other than assessment of glycemic control in a retrospective period [4]. Although relationship between diabetic atherogenesis and several common risk factors (e.g., hyperglycemia, hypertension and hyperlipidemia) have been established [5], few clinical studies have been made, which take glycated products into account as risk factors to predict and evaluate diabetic vascular complications. The aim of this study was to determine the clinical value of serum glycated albumin and high-sensitivity Creactive protein (hs-CRP) levels for the prediction of CAD using logistic regression model in patients with type 2 diabetes.

\section{Methods}

\section{Patients and controls}

The study population consisted of 324 consecutive patients with type 2 diabetes, who underwent coronary angiography for the diagnosis and interventional treatment of CAD in the Department of Cardiology and Department of Endocrinology and Metabolic Diseases, Rui Jin Hospital, Shanghai. The diagnosis of type 2 diabetes was made according to the criteria using a fasting glucose level $\geq 7.0 \mathrm{mmol} / \mathrm{L}$ or 2-hour postprandial glucose level $\geq 11.1 \mathrm{mmol} / \mathrm{L}$. Patients who had a clinical history of type 2 diabetes and were receiving oral hypoglycemic or parental insulin medications were also eligible for the study, but those with type 1 diabetes were excluded by measurement of serum C-peptide and insulin levels. The protocol was approved by the hospital Ethics Committee and written informed consent was obtained from all patients.

\section{Coronary angiography}

Coronary arteriography was performed using standard Judkins techniques or through radial approach. Angiographic analysis was carried out by interventional cardiologists, who were blinded to the study protocol. Mild CAD on visual interpretation was defined as lumen diameter narrowing $<50 \%$, and significant CAD as the presence of any luminal stenosis $\geq 50 \%$. For the purpose of the study, patients were divided into two groups based on visual angiographic results. Control group consisted of 83 patients without any or with mild CAD, and CAD group comprised 241 patients with $\geq 1$ significantly affected coronary artery.

\section{Biochemical investigations}

Blood samples were collected after overnight fasting and stored at $-70^{\circ} \mathrm{C}$ prior to analysis. Serum total cholesterol and triglyceride levels were measured by automated enzymatic procedures (Hoffman-La Roche, Basel, Switzerland). The low-density lipoprotein cholesterol (LDL-C), high-density lipoprotein cholesterol (HDL-C) and lipoprotein (a) (Lp [a]) were determined after separating the lipoprotein fractions from fresh fasting sera by sequential ultracentrifugation. Concentration of apolipoproteins A (apo-A) and B (apo-B) were measured by immunoturbidimetric methods using commercial kit (Boehringer-Mannheim, Mannheim, Germany). Blood urea nitrogen, creatinine, and uric acid were assessed using standard methods.

Serum glycated albumin level was measured with improved bromocresol purple method using Lucica TM glycated albumin-L assay kit (Asahi Kasei Pharma, Japan). Its linear range was $3.2-68.1 \%$ and a maximum interassay coefficient of variation (CV) was $3.0 \%$. The hs-CRP level was determined using a high-sensitivity ELISA kit (Biocheck Laboratories, USA) with linear range of 0.62$119.3 \mathrm{mg} / \mathrm{L}$ and inter-assay CV $<7.5 \%$.

\section{Statistics}

All statistical analyses were performed using SPSS for Windows 13.0 (SPSS Inc., Chicago, Illinois). Data are presented as frequencies and percentages for categorical variables and mean \pm SD for continuous variables, unless otherwise indicated. Differences between groups were assessed using the Chi-square and unpaired tests. Because glycated albumin, triglycerides, hs-CRP, Lp (a), and creatinine values were not normally distributed, between-group differences were assessed by the MannWhitney $U$ test. Univariate analysis was performed to determine each variable's ability in predicting CAD in patients with type 2 diabetes. Variables found to be predictive of CAD in univariate analysis were then entered into a multivariable model using logistic regression to determine the power of each variable for predicting CAD in patients with type 2 diabetes. At the last step of the analysis, the Hosmer-Lemeshow test was use to examine models' goodness of fit. The predictive values of glycated albumin and hs-CRP in the logistic regression model were calculated by constructing receiver-operating characteristic (ROC) curves. A 2-tailed P value of $<0.05$ was considered statistically significant. 


\section{Results \\ Baseline clinical characteristics and biochemical measurements}

Baseline clinical characteristics and biochemical measurements in both groups are listed in Table 1. Patients in $\mathrm{CAD}$ group were older and more male gender and cigarette smoking than those in control group. Blood pressure, serum glucose level and lipid profile were similar in the two groups, except higher serum Lp (a) and reduced apo-A in CAD group. Serum blood urea nitrogen and creatinine levels were significantly higher in CAD group than in control group, indicating reduced renal function in CAD group.

\section{Logistic regression model}

Serum glycated albumin and hs-CRP levels were significantly increased in diabetic patients with CAD than in controls (Table 1). Multivariate regression analysis revealed that older age, male gender, serum concentrations of glycated albumin, hs-CRP, Lp (a), and creatinine were independent risk factors for CAD development in patients with type 2 diabetes (Tables 2 and 3 ). The logistic regression model for predicting $\mathrm{CAD}$ in diabetes was defined as: $\mathrm{P} /(1-\mathrm{P})=\mathrm{EXP}(-1.5+1.265$ gender +0.812 age $+1.24 \mathrm{GA}+0.953$ hs-CRP + $0.902 \mathrm{Lp}[\mathrm{a}]+1.918$ creatinine), and the probability value for each patient was then calculated by equation: $\mathrm{P}=\mathrm{e}^{\mathrm{y}} / 1+\mathrm{e}^{\mathrm{y}}$, where $\mathrm{y}=\ln [\mathrm{P} /(1-\mathrm{P})]$. Hosmer-Lemeshow test was used to check the models' goodness of fit and the results demonstrated a good fit achieved (goodness of fit: Hosmer-Lemeshow $\chi^{2}=7.465$, d.f. $=8, P=0.487)$. Nagelkerke $\mathrm{R}^{2}$ value was 0.35 , suggest- ing the model explained $35 \%$ of the variation in the dependent variable.

\section{Receiver-operating characteristic curves}

ROC plot was calculated to test predictive value of glycated albumin and hs-CRP, and the effectiveness of logistic regression model was also evaluated by constructing ROC plot. A larger area under the curve of hs-CRP was observed compared to glycated albumin. The optimal value of cut-off point for hs-CRP and probability value of regression model to predict CAD in patients with type 2 diabetes were $5.2 \mathrm{mg} / \mathrm{l}$ and 0.648 , respectively. When using hs-CRP as a single predictor, 67 out of 241 diabetic patients with CAD (27.8\%) could be missed (sensitivity $72.2 \%$, specificity $60.0 \%$ ). The use of regression model may cause 43 out of 241 CAD cases (17.7\%) missed (sensitivity $82.3 \%$, specificity $68.6 \%$ ). The ROC plot showed that the optimal cut-off point of regression model for diagnosis of CAD was 0.865 , with only 6 out of 83 cases (7.1\%) without CAD being falsely recognized as CAD (sensitivity 54.5\%, specificity 92.9\%) (Figure 1, Table 4).

\section{Discussion}

Diabetes has been recognized as an important risk factor for CAD, and diabetic patients are at 2-fold increased risk of cardiovascular mortality compared to their nondiabetic counterparts [6]. Previous studies have demonstrated that silent myocardial ischemia, which is mainly caused by autonomic neural dysfunction, occurred in about $20 \%$ to $25 \%$ of diabetic patients, and the prevalence may be as high as $60 \%$ in those at high-risk [7]. Because silent myo-

Table I: Baseline characteristics and biochemical assessments

\begin{tabular}{|c|c|c|c|}
\hline Variable & Control group $(n=83)$ & CAD group $(n=24 I)$ & $P$ value \\
\hline Men (\%) & $38(45.8)$ & $165(68.5)$ & $<0.0001$ \\
\hline Age (yrs) & $62 \pm 10$ & $66 \pm 10$ & 0.001 \\
\hline Cigarette smoking (\%) & II (I3.3) & $68(28.2)$ & 0.007 \\
\hline Hypertension (\%) & $52(62.7)$ & $175(72.6)$ & NS \\
\hline \multicolumn{4}{|l|}{ Blood pressure } \\
\hline Systolic (mmHg) & $138 \pm 21$ & $137 \pm 20$ & NS \\
\hline Diastolic $(\mathrm{mmHg})$ & $81 \pm 10$ & $79 \pm 11$ & NS \\
\hline Hyperlipidemia (\%) & $47(56.6)$ & $137(56.8)$ & NS \\
\hline \multicolumn{4}{|l|}{ Cholesterol } \\
\hline Total cholesterol (mmol/L) & $4.77 \pm 1.27$ & $4.70 \pm 1.15$ & NS \\
\hline HDL-cholesterol (mmol/L) & $1.19 \pm 0.30$ & $1.13 \pm 0.4 \mid$ & NS \\
\hline LDL-cholesterol (mmol/L) & $2.80 \pm 0.89$ & $2.73 \pm 0.90$ & NS \\
\hline Triglycerides(mmol/L) & $2.23 \pm 1.79$ & $2.01 \pm 1.19$ & NS \\
\hline Lipoprotein-a $(g / L)$ & $0.19 \pm 0.11$ & $0.25 \pm 0.19$ & 0.025 \\
\hline Apo-A (g/L) & $1.29 \pm 0.19$ & $1.24 \pm 0.22$ & NS \\
\hline Apo-B $(g / L)$ & $0.94 \pm 0.26$ & $0.94 \pm 0.25$ & NS \\
\hline Glucose (mmol/L) & $7.03 \pm 1.88$ & $7.28 \pm 2.72$ & NS \\
\hline Blood urea nitrogen $(\mathrm{mmol} / \mathrm{L})$ & $5.49 \pm 1.60$ & $6.05 \pm 2.29$ & 0.043 \\
\hline Creatinine $(\mu \mathrm{mol} / \mathrm{L})$ & $74.67 \pm 16.31$ & $92.59 \pm 29.22$ & $<0.0001$ \\
\hline Uric acid $(\mu \mathrm{mol} / \mathrm{L})$ & $307.9 \pm 76.03$ & $314.19 \pm 83.07$ & NS \\
\hline Glycated albumin (\%) & $19.37 \pm 4.29$ & $21.21 \pm 5.24$ & 0.001 \\
\hline $\mathrm{Hs}-\mathrm{CRP}(\mathrm{mg} / \mathrm{L})$ & $7.37 \pm 8.71$ & $25.77 \pm 30.35$ & $<0.0001$ \\
\hline
\end{tabular}


Table 2: Univariate predicators of coronary artery disease in patients with type 2 diabetes

\begin{tabular}{lccc}
\hline Variable & Odds Ratio & 95\% Confidence interval & P Value \\
\hline Men & 3.573 & $1.64-7.81$ & 0.001 \\
Age $\geq 65$ Yrs & 2.267 & $1.12-4.57$ & 0.022 \\
Cigarette smoking & 1.026 & $0.4-2.63$ & 0.958 \\
Hypertension & 1.158 & $0.57-2.36$ & 0.686 \\
Hyperlipidemia & 1.252 & $0.59-2.64$ & 0.556 \\
Total cholesterol & 1.139 & $0.53-2.44$ & 0.737 \\
HDL-cholesterol & 0.871 & $0.57-1.34$ & 0.568 \\
LDL-cholesterol & 0.937 & $0.38-2.29$ & 0.887 \\
Triglycerides & 1.017 & $0.71-1.46$ & 0.927 \\
Lipoprotein-a $\geq 0.22$ g/L & 2.057 & $1.03-4.09$ & 0.04 \\
Blood urea nitrogen & 0.955 & $0.80-1.15$ & 0.624 \\
Creatinine $\geq 100(\mu \mathrm{mol} / \mathrm{L})$ & 8.109 & $2.06-31.9$ & 0.003 \\
Uric acid & $\mathrm{I}$ & $1.00-1.01$ & 0.849 \\
Glycated albumin $\geq 20 \%$ & 3.54 & $1.74-7.20$ & $<0.001$ \\
Hs-CRP $\geq 10 \mathrm{mg} / \mathrm{L}$ & 2.648 & $1.32-5.30$ & 0.006
\end{tabular}

cardial ischemia is responsible for more delayed diagnosis of CAD and poorer prognosis than anginal episodes, early detection and routine screening of CAD with simple tool becomes important and desirable for diabetic population $[1,6,8]$. The current study indicates that several serum biomarkers could be predictive for CAD in patients with type 2 diabetes, among which serum levels of hs-CRP and glycated albumin appeared to be useful in clinical practice.

\section{Serum $L p(a)$ concentration is an independent risk factor for CAD in patients with type 2 diabetes}

Numerous studies have shown that elevated serum level of Lp (a) is an independent risk factor for CAD in nondiabetic subjects, but its contributions to atherosclerosis in diabetes remains in controversy [9-12]. In the present study, Lp (a) was found to be an independent risk factor for CAD development in a diabetic cohort, while HDL-C, LDL-C, total cholesterol, triglyceride, hypertension, and cigarette smoking did not enter this model, suggesting that these traditional risk factors were not risk factors for CAD development in these diabetic subjects. However, we do not believe that the impact of these risk factors can be excluded because of their being quantitatively not different from those in control group or within normal range, compared to $\mathrm{Lp}(\mathrm{a})$. They may exert detrimental effects depending on how much they become glycated and oxidized, which is not easy to determine at present. Cumulative evidence indicates that qualitative changes in lipoproteins may contribute to diabetic angiopathy. Glycated and/or oxidized LDL have demonstrated adverse effects on vascular cell viability, lipid accumulation, growth factor expression and intracellular oxidative stress [13]. Modification of HDL in diabetes by glycation and oxidation may ameliorate efficacy of its vasoprotective functions [13]. In addition, majority of the diabetic patients with CAD in our study received medication of statins, and Lp (a) is less sensitive to statin treatment, compared to other lipoproteins. This may partly explain why $\operatorname{Lp}(\mathrm{a})$ level was higher and entered the model. In this study, serum creatinine level was significantly related to the presence of $\mathrm{CAD}$, and this is consistent with previous findings that patients with renal dysfunction had high incidence of cardiovascular disease, particular in those with late stage renal insufficiency $[14,15]$.

\section{Serum glycated albumin and hs-CRP predict CAD development in patients with type 2 diabetes}

Glycated albumin is a kind of early-stage amadori-modified reaction products formed from Schiff's base adducts [16], and has been implicated in the pathogenesis of diabetic complications $[17,18]$. Previous studies have dem-

Table 3: Multivariate predictors of coronary artery disease in patients with type $\mathbf{2}$ diabetes

\begin{tabular}{lcccc}
\hline Variable & B & Odds ratio & 95\% Cl & P value \\
\hline Constant & -1.5 & 0.223 & & $<0.0001$ \\
Men & 1.265 & 3.542 & $1.818-6.689$ & $<0.0001$ \\
Age $\geq 65$ Yrs & 0.812 & 2.253 & $1.161-4.372$ & 0.016 \\
Glycated albumin $\geq 20 \%$ & 1.24 & 3.456 & $1.777-6.720$ & $<0.0001$ \\
Hs-CRP $\geq 10(\mathrm{mg} / \mathrm{L})$ & 0.953 & 2.593 & $1.313-5.119$ & 0.006 \\
LP $(\mathrm{a}) \geq 0.22 \mathrm{~g} / \mathrm{L}$ & 0.902 & 2.464 & $1.249-4.858$ & 0.009 \\
Creatinine $\geq 100$ umol/L & 1.918 & 6.805 & $1.956-23.673$ & 0.003 \\
\hline
\end{tabular}




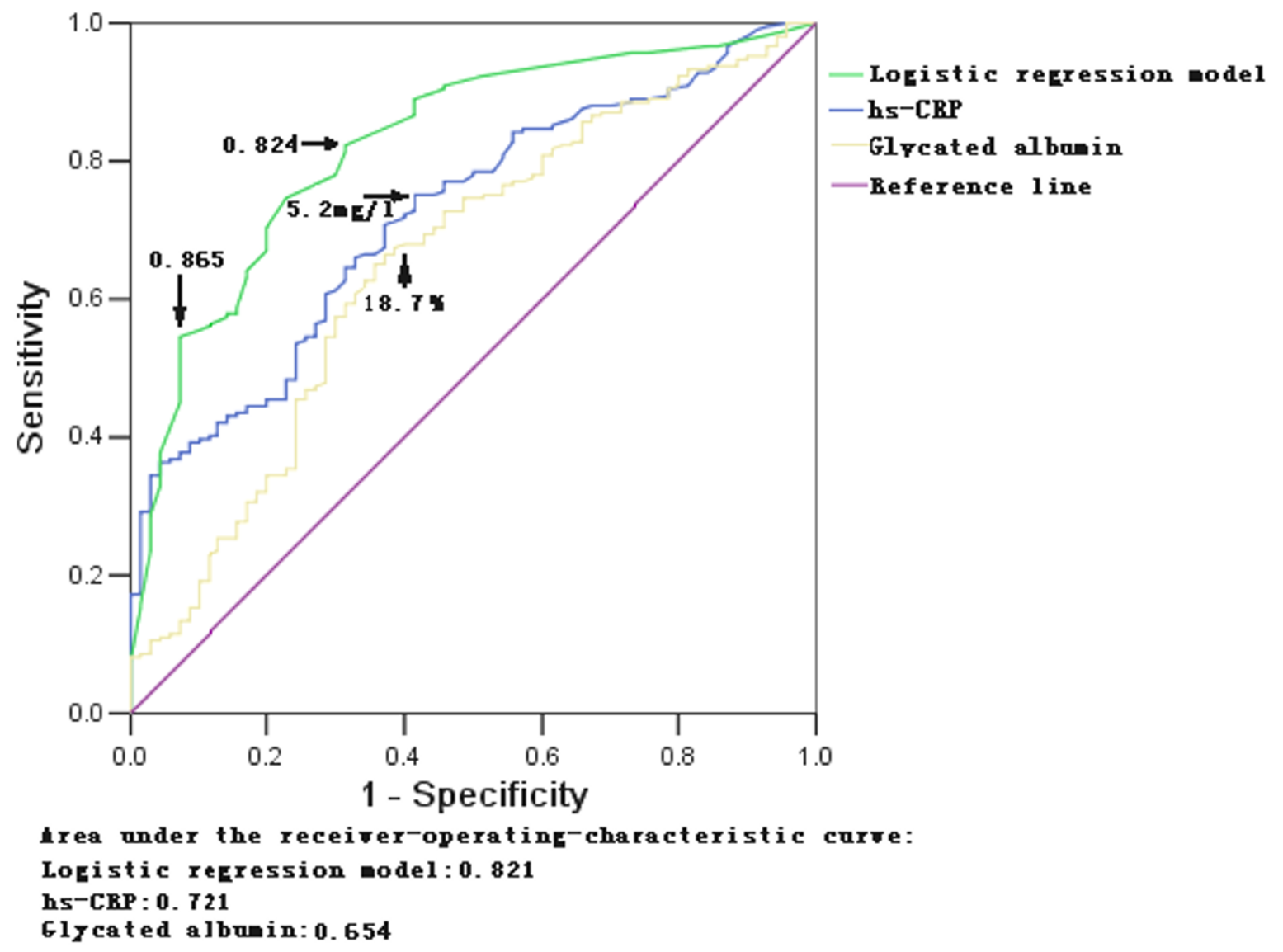

Figure I

ROC curve for glycated albumin, hs-CRP and logistic regression model for predicting coronary artery disease in patients with type 2 diabetes.

onstrated that glycated albumin induces oxidative stress in the vessel wall [19], enhances pro-inflammatory endothelial response to S100A8/A9 [20], and promotes proliferation and migration of vascular smooth muscle cells [21], and thereby is associated with accelerated atherosclerosis. The present study showed that glycated albumin was an independent risk factor for CAD in patients with type 2 diabetes, with odds ratio being 3.456 (95\% CI $1.78-6.72, \mathrm{P}<0.001)$.

Measurement of blood HbA1c level has been widely used to evaluate glycemic control in diabetic patients. We previously observed a significant correlation between serum glycated albumin level and HbA1c concentration ( $\mathrm{r}=$ 0.743, $\mathrm{P}<0.001$, unpublished data). Previous studies have further shown that serum glycated albumin level plateaued several weeks in diabetic patients in whom serum glucose levels were initially poorly controlled and then intensive insulin management was used to rapidly bring glucose levels under control, while serum HbA1c levels were still falling [6]. Therefore, these results indicate that determination of serum glycated albumin level appears to be a useful marker for predicting the presence of diabetic complications, and also a valuable adjunct to HbA1c measurement in reflecting short-term glycemic control.

Table 4: Diagnostic performance of glycated albumin, hs-CRP and logistic regression model

\begin{tabular}{|c|c|c|c|c|c|}
\hline Variable & Area under curve $(95 \% \mathrm{Cl})$ & $P$ value & Cut-off point & Sensitivity & Specificity \\
\hline Glycated albumin & $0.654(0.579-0.730)$ & $<0.0001$ & $18.7 \%$ & $67.9 \%$ & $60 \%$ \\
\hline Hs-CRP & $0.72 \mathrm{I}(0.658-0.785)$ & $<0.0001$ & $5.2 \mathrm{mg} / \mathrm{L}$ & $72.2 \%$ & $60 \%$ \\
\hline Regression model & $0.824(0.768-0.879)$ & $<0.0001$ & 0.648 & $82.3 \%$ & $68.6 \%$ \\
\hline
\end{tabular}


Atherosclerosis is a long-term and chronic inflammatory process that is exacerbated in patients with diabetes $[22,23]$. CRP has been shown to be associated with arteriosclerosis and acute cardiovascular events [24], and has been widely used to stratify patients at high-risk for acute coronary events. Researches showed that patients at intermediate or high risk of CAD may benefit from measurement of hs-CRP with regard to individual risk prediction [25]. The current study revealed that serum level of hs$\mathrm{CRP} \geq 10 \mathrm{mg} / \mathrm{L}$ was associated with a 2.593 -fold increase in risk of CAD in type 2 diabetes when compared with hsCRP level $<10 \mathrm{mg} / \mathrm{L}$. It has been found that good glycemic control per se did not affect nontraditional risk factors for CAD equally despite improved serum glucose level [26], and increased hs-CRP levels were associated with other indicators of diabetes-related cardiovascular risk, but had no correlation with disease duration or glucose control $[27,28]$. These results indicates that serum levels of hsCRP reflect inflammatory status in type 2 diabetes regardless of glycemic control, which merits hs-CRP to be an independent biomarker for predicting CAD. Aggressive intervention of AGEs and inflammatory process is crucial for preventing macrovascular complications in patients with type 2 diabetes, and many agents have been shown to reduce hs-CRP concentrations and to inhibit AGEs formation in these patients $[22,25,29]$.

\section{Predictive value of logistic regression model}

Another major finding of the present study was that the use of logistic regression model incorporating with glycated albumin, hs-CRP and other major independent risk factors commonly seen in type 2 diabetes was competent to screen and predict CAD in patients with diabetes (area under curve: 0.824 ). The optimal probability value of 0.648 may be more suitable for screening CAD in this cohort with a sensitivity of $82.3 \%$ and a specificity of $68.6 \%$. Furthermore, the probability value 0.865 was particularly useful because of its high specificity (92.9\%). Previous study showed that positive predictive value of ECG exercise stress test for angiographically-documented CAD in patients with diabetes was 73\% [6]. Our results suggest that the regression mode may be as effective as exercise stress tests for screening CAD in clinical practice with low cost, and it could also provide useful information regarding the evaluation of the effects of therapeutic modalities aiming to reduce incidence of cardiovascular events in type 2 diabetes.

\section{Limitations}

The present study has several limitations. First, this is in fact a retrospective study and the sample size in the control group was relatively small. Further study is required to increase sample size to ascertain the differences in biomarkers and biochemical measurements between diabetic patients with CAD and controls. As follow-up of the patients has been undergoing and therefore our logistic regression model of CAD prediction in diabetes could be tested by clinical data regarding patient's outcome and disease progression. Secondly, hs-CRP was the only inflammatory factor measured in this study. Whether other "noxious" pro-atherosclerosis inflammatory factors, such as interleukin-6, play a role in the development of $\mathrm{CAD}$ in this cohort remains to be answered. As suggested in some studies, a cluster of "harmful" interleukins exert synergistic and convergent effects on cardiovascular diseases and glucose metabolic alterations [30]. Determination of interleukin helps understand the mechanisms of cardiovascular diabetology on disease progression and thereby provides potential guidance to simultaneously control both atherosclerosis and diabetes. Finally, the Nagelkerke $\mathrm{R}^{2}$ value of the regression model was only 0.350 , suggesting that the model only explained $35 \%$ of variation in the dependent variable, which leaves space for some other pro-atherosclerosis risk factors to complete. Among these possible factors, soluble receptor of AGEs and other factors associated with AGE-receptor system may warrant further investigation.

\section{Conclusion}

Serum glycated albumin and hs-CRP levels were elevated in diabetic patients with CAD. The logistic regression model incorporating with glycated albumin, hs-CRP and other major risk factors of atherosclerosis may be useful for screening CAD in patients with type 2 diabetes.

\section{Abbreviations}

High-density lipoprotein (HDL), low-density lipoprotein (LDL); lipoprotein a [Lp (a)]

\section{Competing interests}

The author(s) declare that they have no competing interests.

\section{Authors' contributions}

LJP collected the samples and performed the experiments, LL designed the study and participated in the whole process of experiments; XWX, RYZ, QZ, JSZ, JH, ZKYang, FHD, QJC, SL, JS and DHF participated or were partly involved in this work. WFS was responsible for the whole research project and was involved in the whole process of the study in its design and coordination, and preparation of the manuscript. All authors read and approved the final manuscript.

\section{Acknowledgements}

This work was supported by a key grant from Shanghai Science and Technology Foundation.

\section{References}

I. Zellweger MJ: Prognostic Significance of Silent Coronary Artery Disease in Type 2 Diabetes. Herz 2006, 3 I:240-245. 
2. Jandeleit-Dahm KA, Lassila M, Allen T): Advanced glycation end products in diabetes-associated atherosclerosis and renal disease: interventional studies. Ann NY Acad Sci 2005, I 043:759-766.

3. Pertynska-Marczewska M, Kiriakidis S, Wait R, Beech J, Feldmann M, Paleolog EM: Advanced glycation end products upregulate angiogenic and pro-inflammatory cytokine production in human monocyte/macrophages. Cytokine 2004, 28:35-47.

4. Rendell M, Paulsen R, Eastberg S, Stephen PM, Valentine JL, Smith CH, Nierenberg J, Rasbold K, Klenk D, Smith PK: Clinical use and time relationship of changes in affinity measurement of glycosylated albumin and glycosylated hemoglobin. Am J Med Sci 1986, 292: | I-I4.

5. Consensus development conference on the diagnosis of coronary artery disease in people with diabetes. In Diabetes Care Volume 21. American Diabetes Association; 1998:155I-1559.

6. Fornengo P, Bosio A, Epifani G, Pallisco O, Mancuso A, Pascale C: Prevalence of silent myocardial ischaemia in new-onset middle-aged Type 2 diabetic patients without other cardiovascular risk factors. Diabet Med 2006, 23:775-779.

7. Scholte AJ, Bax JJ, Wackers FJ: Screening of asymptomatic patients with type 2 diabetes mellitus for silent coronary artery disease: combined use of stress myocardial perfusion imaging and coronary calcium scoring. J Nucl Cardiol 2006, 13:11-18.

8. Kharlip J, Naglieri R, Mitchell BD, Ryan KA, Donner TW: Screening for silent coronary heart disease in type 2 diabetes: clinical application of American Diabetes Association guidelines. Diabetes Care 2006, 29:692-694.

9. Saely CH, Koch L, Schmid F, Marte T, Aczel S, Langer P, Hoefle G, Drexel $\mathrm{H}$ : Lipoprotein(a), type 2 diabetes and vascular risk in coronary patients. Eur J Clin Invest 2006, 36:91-97.

10. Mohan V, Deepa R, Haranath SP, Premalatha G, Rema M, Sastry NG, Enas EA: Lipoprotein(a) is an independent risk factor for coronary artery disease in NIDDM patients in South India. Diabetes Care 1998, $21: 1819-1823$.

II. O'Brien T, Nguyen TT, Harrison JM, Bailey KR, Dyck PJ, Kottke BA: Lipids and $L p(a)$ lipoprotein levels and coronary artery disease in subjects with non-insulin-dependent diabetes mellitus. Mayo Clin Proc 1994, 69:430-435.

12. Pedreno J, Fernandez R, Ballester A, Jornet A, Uson M, Canela J, Petit M: Lack of association of serum lipoprotein (a) levels with type-2 diabetes mellitus in patients with angiographically defined coronary artery disease. Int J Cardiol 2000, 74:I59-167.

13. Jernkins AJ, Best JD, Klein RL, Lyon TJ: Lipoproteins, glycoxidation and diabetic angiopathy. Diabetes Metab Res Rev 2004, 20:349-368.

14. Varma R, Garrick R, McClung J, Frishman WH: Chronic renal dysfunction as an independent risk factor for the development of cardiovascular disease. Cardiol Rev 2005, 13:98-107.

15. Zhang RY, Ni JW, Zhang JS, Hu J, Yang ZK, Zhang Q, Lu AK, Shen WF: Long term clinical outcomes in patients with moderate renal insufficiency undergoing stent based percutaneous coronary intervention. Chin Med J 2006, I I9: I I76-II8I.

16. Higai K, Shimamura A, Matsumoto K: Amadori-modified glycated albumin predominantly induces E-selectin expression on human umbilical vein endothelial cells through NADPH oxidase activation. Clin Chim Acta 2006, 367:137-143

17. Cohen MP, Ziyadeh FN, Chen S: Amadori-modified glycated serum proteins and accelerated atherosclerosis in diabetes: pathogenic and therapeutic implications. J Lab Clin Med 2006, 147:2II-219.

18. Cohen MP: Intervention strategies to prevent pathogenetic effects of glycated albumin. Arch Biochem Biophy 2003, 4I 9:25-30.

19. Liu SX, Hou FF, Guo ZJ, Nagai R, Zhang WR, Liu ZQ, Zhou ZM, Zhou $M$, Xie $D$, Wang GB, Zhang $X$ : Advanced oxidation protein products accelerate atherosclerosis through promoting oxidative stress and inflammation. Arterioscler Thromb Vasc Biol 2006, 26:1156-1162

20. Ehlermann P, Eggers K, Bierhaus A, Most P, Weichenhan D, Greten J, Nawroth PP, Katus HA, Remppis A: Increased proinflammatory endothelial response to SI00A8/A9 after preactivation through advanced glycation end products. Cardiovasc Diabetol 2006, 5:6.
21. Hattori Y, Suzuki M, Hattori S, Kasai K: Vascular smooth muscle cell activation by glycated albumin (amadori adducts). Hypertension 2002, 39:22-28.

22. Muhlestein JB, May HT, Jensen JR, Horne BD, Lanman RB, Lavasani $F$, Wolfert RL, Pearson RR, Yannicelli HD, Anderson JL: The reduction of inflammatory biomarkers by statin, fibrate, and combination therapy among diabetic patients with mixed dyslipidemia: the DIACOR (Diabetes and Combined Lipid Therapy Regimen) study. J Am Coll Cardiol 2006, 48:396-40I.

23. Ross R: Atherosclerosis-an inflammatory disease. $N$ EnglJ Med 1999, 340: II5-126.

24. Wilson AM, Ryan MC, Boyle Al: The novel role of C-reactive protein in cardiovascular disease: risk marker or pathogen. Int J Cardiol 2006, 106:29|-297.

25. Pfutzner A, Forst T: High-sensitivity C-reactive protein as cardiovascular risk marker in patients with diabetes mellitus. Diabetes Technol Ther 2006, 8:28-36.

26. Fonseca VA, Theuma P, Mudaliar S, Leissinger CA, Clejan S, Henry RR: Diabetes treatments have differential effects on nontraditional cardiovascular risk factors. J Diabetes Complications 2006, 20: 14-20.

27. Kimberly MM, Cooper GR, Myers GL: An overview of inflammatory markers in type 2 diabetes from the perspective of the clinical chemist. Diabetes Technol Ther 2006, 8:37-44.

28. Pfutzner A, Standl E, Strotmann HJ, Schulze J, Hohberg C, Lubben G, Pahler S, Schondorf T, Forst T: Association of high-sensitive Creactive protein with advanced stage beta-cell dysfunction and insulin resistance in patients with type 2 diabetes mellitus. Clin Chem Lab Med 2006, 44:556-560.

29. Myint KM, Yamamoto Y, Sakurai S, Harashima A, Watanabe T, Li H, Takeuchi A, Yoshimura K, Yonekura H, Yamamoto H: Blockade of diabetic vascular injury by controlling of AGE-RAGE system. Curr Drug Targets 2005, 6:447-452.

30. Fisman EZ, Motro M, Tenenbaum A: Cardiovascular diabetology in the core of a novel interleukins classification: the bad, the good and the aloof. Cardiovasc Diabetol 2003, 2: II.

Publish with Bio Med Central and every scientist can read your work free of charge

"BioMed Central will be the most significant development for disseminating the results of biomedical research in our lifetime. "

Sir Paul Nurse, Cancer Research UK

Your research papers will be:

- available free of charge to the entire biomedical community

- peer reviewed and published immediately upon acceptance

- cited in PubMed and archived on PubMed Central

- yours - you keep the copyright 